Arch. Math. 86 (2006) 97-100

0003-889X/06/020097-04

DOI 10.1007/s00013-005-1484-x

(C) Birkhäuser Verlag, Basel, 2006

Archiv der Mathematik

\title{
Abelian groups determined by subgroup lattices of direct powers
}

\author{
By \\ GRIGORE CÁLugĂREANU
}

\begin{abstract}
In this short note, we show that the class of abelian groups determined by the subgroup lattice of their direct $n$-powers is exactly the class of the abelian groups which share the $n$-root property. As applications we answer in the negative a (semi)conjecture of Pálfy and solve a more general problem.
\end{abstract}

Recently, for an arbitrary group $G$, the subgroup lattice of the square $G \times G$ has received some attention (see [10] and [13]) with respect to the following

Problem. If the direct squares of two groups are projective (i.e., have isomorphic subgroup lattices), are these groups isomorphic? In other words, does the subgroup lattice of the direct square determine the group up to an isomorphism?

This problem has negative answer: the A. Rottlaender groups (see [11]). However, for simple groups (see [14]) and for finite abelian groups (see [9]) the answer is affirmative.

Let $\mathcal{S}_{2}$ be the class of the abelian groups which share the square root property (i.e., $G \in \mathcal{S}_{2}$ iff $G \cong H$ whenever $\left.G \oplus G \cong H \oplus H\right)$ and $\mathcal{P}_{2}$ the class of the abelian groups which are determined by the subgroup lattice of their direct squares (i.e., $G \in \mathcal{P}_{2}$ iff $G \cong H$ whenever $L(G \oplus G) \cong L(H \oplus H)$, lattice isomorphism; here $L(G)$ denotes the subgroup lattice of the group $G)$. For notation and terminology we refer to [5].

Main result. $\mathcal{S}_{2}=\mathcal{P}_{2}$.

Theorem 1. An abelian group is determined by the subgroup lattice of its direct square if and only if it has the square-root property.

Mathematics Subject Classification (2000): 20K27; 20D30; 06B25.

This work was supported by Kuwait University Research Administration Grant No. SM 04/04. 
Proof. Actually, more can be proved: let $G$ be an abelian group and $H$ an arbitrary group. $G \times G$ is projective to $H \times H$ if and only if $G \times G$ and $H \times H$ are isomorphic.

If $G$ is abelian, so is $G \times G$. Hence $L(G \times G)$ is modular and so is $L(H \times H)$. Using a result of Lukács-Pálfy, (see [9]), $H$ must be abelian, too.

If $G$ is not torsion then the torsion-free rank $r_{0}(G \times G)$ is at least 2. Since $G \times G$ is projective to $H \times H$, by an early result of Baer (see [3]), $G \times G$ and $H \times H$ are isomorphic.

If $G$ is torsion then the $p$-rank $r_{p}(G \times G)$ is at least 2 for every $p$ such that the primary component $G_{p} \neq 0$. Again, since $G \times G$ is projective to $H \times H$, by a similar result due to Fuchs (see [5, § 80]), $G \times G \cong H \times H$.

For every positive integer $n \geqq 2$, the same ingredients are needed in order to show that

Re mark 1. An abelian group is determined by the subgroup lattice of its $n$-th direct power if and only if it has the $n$-th root property (i.e., for abelian groups, $G^{n}$ is projective to $H^{n}$ if and only if $G^{n}$ is isomorphic to $H^{n}$ ).

Ex a m ples. The only class mentioned in the literature as included in $\mathcal{P}_{2}$ is the class of the finite (abelian) groups (see [9]). We can widely improve this: as for now, the following classes of abelian groups are known to share the square-root property:

(i) countable torsion groups (Kaplansky, [8]),

(ii) countable mixed groups of torsion-free rank 1 (Rotman, [12]),

(iii) groups with semilocal endomorphism rings (actually these have the $n$-th root property, for any positive integer $n \geqq 2$ ); these are (see [4]) exactly

- finitely generated, if torsion

- with $p G=G$ for all but a finite prime numbers $p$, if torsion-free of finite rank (groups called semilocal in [2])

- splitting, with both torsion and torsion-free parts having semilocal endomorphism rings, if mixed.

Applications. Our result enables us to translate problems related to projectivities into problems related to decompositions. Pathologies of decompositions for (especially finite rank torsion-free) abelian groups were intensively studied. From [7] (or [6, 90.3]), we recall the following result:

Given any integer $m \geqq 2$, there exist two (nonisomorphic) torsion-free indecomposable groups of rank $2, A$ and $C$ such that $A \oplus A \oplus \ldots \oplus A \cong C \oplus C \oplus \ldots \oplus C$ ( $n$ summands) if and only if $n \equiv 0(\bmod m)$.

First, we answer in the negative a conjecture (almost) made by Pálfy in [10]: "it still may be true that if the subgroup lattices of some power distinguish two groups then already the cubes do". That is, if for some $n \geqq 4$ we have $L\left(G^{n}\right) \varsubsetneqq L\left(H^{n}\right)$ then also $L\left(G^{3}\right) \varsubsetneqq L\left(H^{3}\right)$. 
Equivalently, if $L\left(G^{3}\right) \cong L\left(H^{3}\right)$ then $L\left(G^{n}\right) \cong L\left(H^{n}\right)$. Using our previous remark, this is also equivalent with: if $G^{3} \cong H^{3}$ then also $G^{n} \cong H^{n}$ for all $n \geqq 4$. In order to see that this is false, in the above mentioned result it suffices to take $m=3$ (and $n=3$ and 4): we have $A^{3} \cong C^{3}$ but $A^{4} ¥ C^{4}$.

Finally, we have

Theorem 2. For $m$ and $n$ different positive integers $L\left(G^{m}\right) \cong L\left(H^{m}\right)$ implies $L\left(G^{n}\right) \cong$ $L\left(H^{n}\right)$ for every groups $G$ and $H$ if and only if both $m, n \geqq 2$ and $m$ divides $n$.

Pro of. First suppose $m, n \geqq 2$. By the above remark, equivalently, we have to deal with $G^{m} \cong H^{m}$ implies $G^{n} \cong H^{n}$, obviously true if $m$ divides $n$ (for any positive integer $k$, $G^{m} \cong H^{m}$ implies $\left.G^{k m} \cong H^{k m}\right)$. None of the remaining implications is true: indeed, in the remaining case $n \not \equiv 0(\bmod m)$, so taking $G=A$ and $H=C$, one has $G^{m} \cong H^{m}$ but $G^{n} \approx H^{n}$.

Secondly, consider $m=1$ and $n \geqq 2, G=\mathbb{Q}_{p}=\left\{\frac{m}{n} \in \mathbb{Q} \mid(n ; p)=1\right\}$ and $H=\mathbb{Q}_{q}$ (for different primes $p$ and $q$ ). Since the type of $\mathbb{Q}_{p}$ can be obtained from the type of $\mathbb{Q}_{q}$ by a permutation of the set of all the prime numbers, by a Theorem of Fuchs (see $[5, \mathrm{p}$. 305]), $L\left(\mathbb{Q}_{p}\right) \cong L\left(\mathbb{Q}_{q}\right)$. Further, by the way of contradiction, suppose $L\left(\mathbb{Q}_{p}^{n}\right) \cong L\left(\mathbb{Q}_{q}^{n}\right)$. Once again by the previous remark, this is equivalent to $\mathbb{Q}_{p}^{n} \cong \mathbb{Q}_{q}^{n}$ which implies $\mathbb{Q}_{p} \cong \mathbb{Q}_{q}$ (these two groups are semi-local; therefore they share the $n$-th root property). But this is impossible: these rank 1 torsion-free groups are determined by their types and consequently are not isomorphic.

Finally, suppose $m \geqq 2$ and $n=1$. Once again, $L\left(A^{m}\right) \cong L\left(C^{m}\right)$ (equivalent to $A^{m} \cong C^{m}$ ) but $A \nsubseteq C$. Nor $L(A) \cong L(C)$ is possible, because $A, C$ are torsion-free groups of rank 2 (Baer's result would imply $A \cong C$ ).

R e m a rk 2. In particular, the above result shows that generally, there are no inclusions between $\mathcal{P}_{1}$ the class of the abelian groups which are determined by their subgroup lattice [which is better known; the only exceptions must have torsion-free rank 1] and $\mathcal{P}_{2}$ the class of the abelian groups which are determined by the subgroup lattice of their direct squares, nor between $\mathcal{P}_{2}$ and $\mathcal{P}_{3}$.

Example 2.11 from [1] (which actually comes back to Jónsson), points out two nonisomorphic groups with projective squares, which are not projective.

\section{References}

[1] D. ARNOLD, Finite Rank Torsion-Free Abelian Groups and Rings. LNM 931, Berlin-Heidelberg-New York 1982.

[2] D. ARNOLD, Abelian Groups and Representations of Finite Partially Ordered Sets. CMS Books in Mathematics, New York 2000.

[3] R. BAER, The significance of the system of subgroups for the structure of a group. Amer. J. Math. 71, $1-44$ (1939).

[4] G. CĂLUgăREANU, Abelian groups with semilocal endomorphism rings. Comm. Algebra 30(9), 4105-4111 (2002). 
[5] L. Fuchs, Abelian Groups. Akadémiai Kiadó, Budapest 1958.

[6] L. FuCHS, Infinite Abelian Groups, Vol. II. Pure Appl. Math. 36, 1973.

[7] L. FUCHS and F. LOONSTRA, On direct decompositions of torsion-free abelian groups of finite rank. Rend. Sem. Mat. Univ. Padova 44, 75-83 (1970).

[8] I. KAPLANSKY, Infinite Abelian Groups. Ann Arbor 1954.

[9] E. LUKÁCS and P. P. PÁLFY, Modularity of the subgroup lattice of a direct square. Arch. Math (Basel) 46, 18-19 (1986)

[10] P. P. PÁlfy, Groups and Lattices. In: Proceedings. Groups St Andrews 2001 in Oxford 1, 428-454, Cambridge 2003.

[11] A. RotTLAENDER, Nachweis der Existenz nicht-isomorpher Gruppen von gleicher Situation der Untergruppen. Math. Z. 28, 641-653 (1928).

[12] J. Rotman, Torsion-free and mixed abelian groups. Illinois J. Math. 5, 131-143 (1961).

[13] R. SCHMIDT, New Results and Methods in the Theory of Subgroup Lattices of Groups. In: Topics in infinite groups, 239-275. Quad. Mat. 8, Aracne, Rome 2001.

[14] M. SUZUKI, On the lattice of subgroups of finite groups. Trans. Amer. Math. Sec. 70, 345-371 (1951).

Received: 24 February 2005

Grigore Călugăreanu

Dept. of Mathematics and Computer Science

Faculty of Science

Kuwait University

Kuwait

calu@sci.kuniv.edu.kw 